\title{
Numerical Solutions of a Quadratic Integral Equations by Using Variational Iteration and Homotopy Perturbation Methods
}

\author{
Hind Al-badrani ${ }^{1}$, F. A. Hendi ${ }^{2} \&$ Wafa Shammakh ${ }^{2}$ \\ ${ }^{1}$ Department of Management Information Systems, Taibah University, Madinah, Saudi Arabia \\ ${ }^{2}$ Department of Mathematics, King Abdulaziz University, Jeddah, Saudi Arabia \\ Correspondence: Hind Al-badrani, Department of Management Information Systems, Taibah University, Madinah, Saudi \\ Arabia. E-mail: hal7rbi@hotmail.com
}

Received: February 7, 2017 Accepted: February 27, 2017 Online Published: March 26, 2017

doi:10.5539/jmr.v9n2p134 URL: https://doi.org/10.5539/jmr.v9n2p134

\begin{abstract}
In this paper, the approximate solutions for quadratic integral equations (QIEs) are given by the variational iteration method (VIM) and homotopy perturbation method (HPM). These methods produce the solutions in terms of convergent series without needing to restrictive assumptions, to illustrate the ability and credibility of the methods, we deal with some examples that show simplicity and effectiveness.
\end{abstract}

Keywords: Quadratic Integral Equations, Variational Iteration Method, Homotopy Perturbation Method.

Subject Code: $45 \mathrm{G} 10$.

\section{1. introduction}

Quadratic integral equations (QIEs) are often applied in the radiative transfer, neutron transport, kinetic theory of gases and in the traffic theories.

The QIEs are studied in many papers and monographs (Bana's, et al., 2005; Bana's, et al., 1998; Bana's \& Martinon, 2004; El-sayed \& Hashem, 2009a; El-Sayed \& Hashem, 2009b).

Recently, the different analytical and numerical methods are applied to reach the approximate solutions of QIEs. As there is no exact solutions for the most QIEs, many different kinds of researches are focusing on the effective of QIEs properties like the existence, uniqueness, positive solutions and monotonic solutions of this class of equations (Argyros, 1985; Bana's et, al., 1998; Bana's \& Martinon, 2004; El-Sayed \& Rzepka, 2006). There are few papers which have dealt with the numerical solutions of QIEs such as Elsayed (El-Sayed et al., 2010) used the classical method of successive approximations Picard and Adomian decomposition method for solving QIEs, Avazzadeh (Avazzadeh, 2012) used the radial basis functions to obtain the approximate solutions of QIEs of Urysohn's type. (He, 1999a; He, 1999b; He, 2000; He, 2003) was the first one who proposed the VIM and HPM to find the solutions of linear and nonlinear problems.

Widely, the VIM is used in the literature in different scientific applications in (Abdou \& Soliman, 2005; Abulwafa et al., 2006; He \& Wu, 2007). This method presents significant enhancements over existing numerical and analytic technique like the perturbation, Adomian, Galerkin, finite differences methods, etc. These methods have dealt with ordinary, partial differential equations, the integro-differential equations (IDEs) and integral equations, in a direct way without needing to any specific restriction which may give the closed form of exact solution if there is an exact solution. The VIM has no specific restrictions for nonlinear terms which involve in the equation.

The homotopy perturbation method deforms a difficult problem under study into a simple one which is easy to solve. Most perturbation methods assume there is a small parameter, but there is no small parameter at all in the most nonlinear problems. Many new methods are proposed to eliminate the small parameter (He, 1999b; Liao, 1995). Also, the HPM is employed for solving several kinds of integral equations. Such as, Fredholm, nonlinear Volterra-Fredholm integral equations and Volterra integro-differential equations.

The aim of the present paper is extending the application of HPM and VIM to give some approximate solutions for the following QIE where $\mathcal{A}(t)$ is given and $\mathcal{F}(s, x(s))$ is any nonlinear functions. We want to point out that this work is applied for first time on these kind of equations.

$$
x(t)=\mathcal{A}(t)+\mathcal{G}(t, x(t)) \int_{0}^{t} \mathcal{F}(s, x(s)) d s .
$$

It is clear that the results reveal the effectively and simplicity for the presented two methods. 


\section{Variational Iteration Method}

Consider the following differential equation where $\mathcal{L}$ and $\mathcal{N}$ are linear and nonlinear operators respectively, and $\mathrm{g}(x)$ is the inhomogeneous source term

$$
\mathcal{L}[\mathrm{u}(x)]+\mathcal{N}[\mathrm{u}(x)]=\mathrm{g}(x) .
$$

The VIM presents a correction functional for eq.(2) in the following form:

$$
\mathrm{u}_{n+1}(x)=\mathrm{u}_{n}(x)+\int_{0}^{x} \lambda(\zeta)\left[\mathcal{L} \mathrm{u}_{n}(\zeta)+\mathcal{N} \widetilde{\mathrm{u}_{n}}(\zeta)-\mathrm{g}(\zeta)\right] d \zeta,
$$

where $\lambda$ is a general Lagrange multiplier, noting that in this method $\lambda$ may be a constant or a function, which can be identified perfectly by the variational theory and the subscript $n$ denotes the nth-order approximation, $\widetilde{\mathrm{u}}_{n}$ is considered as a restricted value that means it behaves as a constant, i.e. $\delta \widetilde{\mathrm{u}_{n}}=0$.

It was found in (Abdou \& Soliman, 2005; Abulwafa et al., 2006; He \& Wu, 2007). the general formula for $\lambda(x)$ for the $n$th order differential equation

$$
\mathbf{u}^{(n)}+f\left(\mathrm{u}(\zeta), \mathbf{u}^{\prime}(\zeta), \mathbf{u}^{\prime \prime}(\zeta), \ldots, \mathrm{u}^{(n)}(\zeta)\right)=0
$$

has the form

$$
\lambda(x)=(-1)^{n} \frac{1}{(n-1) !}(\zeta-x)^{(n-1)} .
$$

The solution given by

$$
\mathrm{u}(x)=\lim _{n \rightarrow \infty} \mathrm{u}_{n}(x)
$$

\section{Homotopy Perturbation Method}

Consider the differential equation (2) with following the boundary conditions where B is a boundary operator, $\Gamma$ is the boundary of the domain $\Omega$ and $x \in \Omega$

$$
\mathrm{B}\left(\mathrm{u}, \frac{\partial \mathrm{u}}{\partial n}\right)=0, \quad x \in \Gamma .
$$

The He's homotopy perturbation technique (He, 1999a), (He, 2000) defines the homotopy $v(x, p): \Omega \times[0,1] \rightarrow \mathfrak{R}$ which satisfies

$$
\mathcal{H}(v, p)=(1-p)\left[\mathcal{L}(v)-\mathcal{L}\left(\mathrm{u}_{0}\right)\right]+p[\mathcal{L}(v)+\mathcal{N}(v)-\mathrm{g}(x)]=0,
$$

or

$$
\mathcal{H}(v, p)=\left[\mathcal{L}(v)-\mathcal{L}\left(\mathrm{u}_{0}\right)\right]+p \mathcal{L}\left(\mathrm{u}_{0}\right)+p[\mathcal{N}(v)-\mathrm{g}(x)]=0,
$$

where $x \in \Omega$ and $p \in[0,1]$ is an impeding parameter, $\mathrm{u}_{0}$ is an initial approximation which satisfies the boundary conditions, from eq's.(7) and (8), we have

$$
\begin{aligned}
& \mathcal{H}(v, 0)=\mathcal{L}(v)-\mathcal{L}\left(\mathrm{u}_{0}\right)=0, \\
& \mathcal{H}(v, 1)=\mathcal{L}(v)+\mathcal{N}(v)-\mathrm{g}(x)=0 .
\end{aligned}
$$

The $p$ process of changing from zero to unity is just that of $v(x, p)$ from $\mathrm{u}_{0}$ to $\mathrm{u}(x)$. In topology, this is called deformation, $\mathcal{L}(v)-\mathcal{L}\left(\mathrm{u}_{0}\right)$ and $\mathcal{L}(v)+\mathcal{N}(v)-\mathrm{g}(x)$ are homotopic. The solutions of eq.(7) and eq.(8) can be defined as a power series in $p$

$$
v=v_{0}+p v_{1}+p^{2} v_{2}+\ldots .
$$

when $p \rightarrow 1$, corresponding to (7) becomes the approximate solution is

$$
\mathrm{u}=v_{0}+v_{1}+v_{2}+\ldots
$$

the convergence of the series (12) has been proved in (He, 1999a; He, 2000). 


\section{Numerical Examples}

In this part, we study some examples and apply the VIM and HPM methods for comparison reasons.

Example 1. solve the QIE (El-Sayed et al., 2010)

$$
x(t)=\left(t^{2}-\frac{t^{10}}{35}\right)+\frac{t}{5} x(t) \int_{0}^{t} s^{2} x^{2}(s) d s
$$

with exact solution $x(t)=t^{2}$.

as beginning we have to convert volterra QIE to an equivalent volterra IDE. We can do this by differentiating two sides of the QIEs, we should used the Leibnitz rule for differentiating the QIEs at the right side.

$$
x^{\prime}(t)=2 t-10\left(\frac{t^{9}}{35}\right)+\frac{t^{3}}{5} x^{3}(t)+\left(\frac{1}{5} x(t)+\frac{t}{5} x^{\prime}(t)\right) \int_{0}^{t} s^{2} x^{2}(s) d s, \quad x(0)=0,
$$

we can get the initial condition $x(0)=0$ by substituting $x=0$ in eq.(13), the correction functional for Equation (14) is

$$
\begin{aligned}
x_{n+1}(t)= & x_{n}(t)+\int_{0}^{t} \lambda(\zeta)\left(x_{n}^{\prime}(\zeta)-2 \zeta+10 \frac{\zeta^{9}}{35}-\frac{\zeta^{3}}{5} x_{n}^{3}(\zeta)-\left(\frac{1}{5} x_{n}(\zeta)+\frac{\zeta}{5} x_{n}^{\prime}(\zeta)\right)\right. \\
& \left.\int_{0}^{\zeta} r^{2} x^{2}(r) d r\right) d \zeta .
\end{aligned}
$$

We substitute the value of $\lambda(\zeta)=-1$ in eq.(15) which is identified by the variational theory, also, we can use the initial value $x(0)=0$ to obtain the zeroth approximation $x_{0}(t)$ and by using Equation (15) we get the successive approximations,

$$
\begin{aligned}
& x_{0}(t)=0 \\
& x_{1}(t)=t^{2}-\frac{1}{35} t^{10}, \\
& x_{2}(t)=t^{2}-\frac{1}{4930625} t^{34}+\frac{61}{2113125} t^{26}-\frac{29}{18375} t^{18},
\end{aligned}
$$

and so on, and the solution given by

$$
x(t)=\lim _{n \rightarrow \infty} x_{n}(t)
$$

Table 1. Comparison of the numerical results with the exact solution $x(t)$

\begin{tabular}{cccc}
\hline$t$ & Approximate Solution & Exact Solution & Absolute error \\
\hline 0.10 & 0.01000000 & 0.01000000 & $3.008 \times 10^{-40}$ \\
0.20 & 0.04000000 & 0.04000000 & $5.168 \times 10^{-30}$ \\
0.30 & 0.09000000 & 0.09000000 & $5.017 \times 10^{-24}$ \\
0.40 & 0.16000000 & 0.16000000 & $8.879 \times 10^{-20}$ \\
0.50 & 0.25000000 & 0.25000000 & $1.751 \times 10^{-16}$ \\
0.60 & 0.36000000 & 0.36000000 & $8.617 \times 10^{-14}$ \\
0.70 & 0.49000000 & 0.49000000 & $1.626 \times 10^{-11}$ \\
0.80 & 0.64000000 & 0.64000000 & $1.521 \times 10^{-9}$ \\
0.90 & 0.80999992 & 0.81000000 & $8.311 \times 10^{-8}$ \\
1.00 & 0.99999704 & 1.00000000 & 0.0000029606 \\
\hline
\end{tabular}




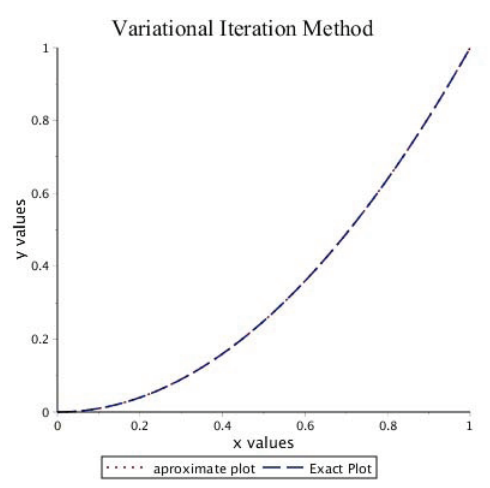

Figure 1. Comparison of the approximate solution by VIM with the exact solution

Table 1 shows the approximate solution for $n=4$, also it is obvious that we can improve the accuracy of solutions by computing more terms of the approximate solutions. We can construct the following homotopy according to HPM,

$$
\mathcal{H}(\mathrm{u}, p)=(1-p)(\mathrm{u}(t)-\mathrm{g}(t))+p\left(\mathrm{u}(t)-\left(t^{2}-\frac{t^{10}}{35}\right)-\frac{t}{5} \mathrm{u}(t) \int_{0}^{t} s^{2} \mathrm{u}^{2}(s) d s\right)=0
$$

such that $\mathrm{g}(t)=\left(t^{2}-\frac{t^{10}}{35}\right)$ then

$$
\mathcal{H}(\mathrm{u}, p)=\mathrm{u}(t)-\left(t^{2}-\frac{t^{10}}{35}\right)-p \frac{t}{5} \mathrm{u}(t) \int_{0}^{t} s^{2} \mathrm{u}^{2}(s) d s=0
$$

substituting (11) into (20) and equating the terms with the same identical powers of $p$ we have

$$
\begin{aligned}
p^{0}: \mathrm{u}_{0}(t)= & \left(t^{2}-\frac{t^{10}}{35}\right) \\
p^{1}: \mathrm{u}_{1}(t)= & \frac{t}{5} \mathrm{u}_{0}(t) \int_{0}^{t} s^{2} H_{0}(s) d s, \\
p^{2}: \mathrm{u}_{2}(t)= & \frac{t}{5} \mathrm{u}_{0}(t) \int_{0}^{t} s^{2} H_{1}(s) d s+\frac{t}{5} \mathrm{u}_{1}(t) \int_{0}^{t} s^{2} H_{0}(s) d s, \\
p^{3}: \mathrm{u}_{3}(t)= & \frac{t}{5} \mathrm{u}_{0}(t) \int_{0}^{t} s^{2} H_{2}(s) d s+\frac{t}{5} \mathrm{u}_{1}(t) \int_{0}^{t} s^{2} H_{1}(s) d s \\
& +\frac{t}{5} \mathrm{u}_{2}(t) \int_{0}^{t} s^{2} H_{0}(s) d s,
\end{aligned}
$$

and so on, where $H_{i}$ are He's polynomials of the nonlinear term $x^{2}$, and the solution will be,

$$
\mathrm{u}(t)=\sum_{i=0}^{n} \mathrm{u}_{i}(t)
$$


Tbale 2. Comparison of the numerical results with the exact solution $x(t)$

\begin{tabular}{cccc}
\hline$t$ & Approximate Solution & Exact Solution & Absolute error \\
\hline 0.10 & 0.01000000 & 0.01000000 & $4.992 \times 10^{-49}$ \\
0.20 & 0.04000000 & 0.04000000 & $2.195 \times 10^{-36}$ \\
0.30 & 0.09000000 & 0.09000000 & $5.462 \times 10^{-29}$ \\
0.40 & 0.16000000 & 0.16000000 & $9.655 \times 10^{-24}$ \\
0.50 & 0.25000000 & 0.25000000 & $1.134 \times 10^{-19}$ \\
0.60 & 0.36000000 & 0.36000000 & $2.398 \times 10^{-16}$ \\
0.70 & 0.49000000 & 0.49000000 & $1.549 \times 10^{-13}$ \\
0.80 & 0.64000000 & 0.64000000 & $4.181 \times 10^{-11}$ \\
0.90 & 0.80999999 & 0.81000000 & $5.745 \times 10^{-9}$ \\
1.00 & 0.99999954 & 1.00000000 & $4.554 \times 10^{-7}$ \\
\hline
\end{tabular}

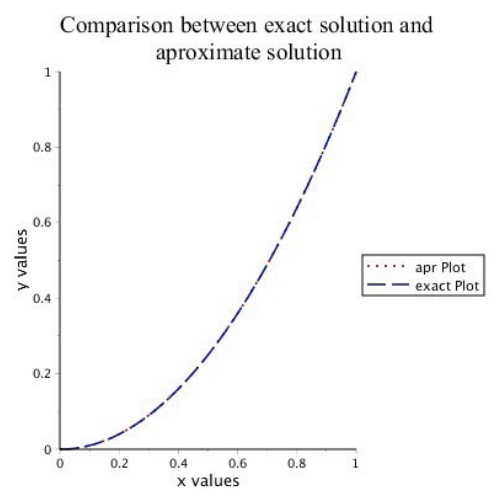

Figure 2. Comparison of the approximate solution by HPM with the exact solution

Table 2 shows the approximate solution for $n=4$, also it is obvious that we can improve the accuracy of solutions by computing more terms of the approximate solutions.

Example 2. Solve the QIE (El-Sayed et al., 2010)

$$
x(t)=\left(t^{3}-\frac{t^{19}}{100}-\frac{t^{20}}{110}\right)+\frac{t^{3}}{10} x^{2}(t) \int_{0}^{t}(s+1) x^{3}(s) d s,
$$

with exact solution $x(t)=t^{3}$.

as beginning we have to convert volterra QIE to an equivalent volterra IDE. We can do this by differentiating two sides of the QIEs, we should used the Leibnitz rule for differentiating the QIEs at the right side.

$$
\begin{aligned}
x^{\prime}(t)= & \left(3 t^{2}-19 \frac{t^{18}}{100}-20 \frac{t^{19}}{110}\right)+3 \frac{t^{2}}{10} x^{2}(t) \int_{0}^{t}(s+1) x^{3}(s) d s+\frac{t^{3}}{10}\left(2 x(t) x^{\prime}(t)\right) \int_{0}^{t}(s+1) x^{3}(s) d s \\
& +\frac{t^{3}}{10}(t+1) x^{5}(t) d s, \quad x(0)=0,
\end{aligned}
$$

we can get the initial condition $x(0)=0$ by substituting $x=0$ in eq.(25), the correction functional for Equation (26) is

$$
\begin{aligned}
x_{n+1}(t)= & x_{n}(t)+\int_{0}^{t} \lambda(\zeta)\left(x_{n}^{\prime}(\zeta)-3 \zeta^{2}+19 \frac{\zeta^{18}}{100}+20 \frac{\zeta^{19}}{110}-3 \frac{\zeta^{2}}{10} x_{n}^{2}(\zeta) \int_{0}^{\zeta}(r+1) x_{n}^{3}(r) d r\right. \\
& \left.-\frac{\zeta^{3}}{10}\left(2 x_{n}(\zeta) x_{n}^{\prime}(\zeta)\right) \int_{0}^{\zeta}(r+1) x_{n}^{3}(r) d r-\frac{\zeta^{3}}{10}(\zeta+1) x_{n}^{5}(\zeta)\right) d \zeta,
\end{aligned}
$$


We substitute the value of $\lambda(\zeta)=-1$ in eq.(27) which is identified by the variational theory, also, we can use the initial value $x(0)=0$ to obtain the zeroth approximation $x_{0}(t)$ and the solution given by

$$
x(t)=\lim _{n \rightarrow \infty} x_{n}(t)
$$

Table 3. Comparison of the numerical results with the exact solution $x(t)$

\begin{tabular}{cccc}
\hline$t$ & Approximate Solution & Exact Solution & Absolute error \\
\hline 0.10 & 0.00100000 & 0.00100000 & $1.112 \times 10^{-56}$ \\
0.20 & 0.00800000 & 0.00800000 & $3.189 \times 10^{-41}$ \\
0.30 & 0.02700000 & 0.02700000 & $3.813 \times 10^{-32}$ \\
0.40 & 0.06400000 & 0.06400000 & $1.105 \times 10^{-25}$ \\
0.50 & 0.12500000 & 0.12500000 & $1.175 \times 10^{-20}$ \\
0.60 & 0.21600000 & 0.21600000 & $1.540 \times 10^{-16}$ \\
0.70 & 0.34300000 & 0.34300000 & $4.749 \times 10^{-13}$ \\
0.80 & 0.51200000 & 0.51200000 & $5.066 \times 10^{-10}$ \\
0.90 & 0.72899976 & 0.72900000 & $2.393 \times 10^{-7}$ \\
1.00 & 0.99994142 & 1.00000000 & 0.0000585810 \\
\hline
\end{tabular}

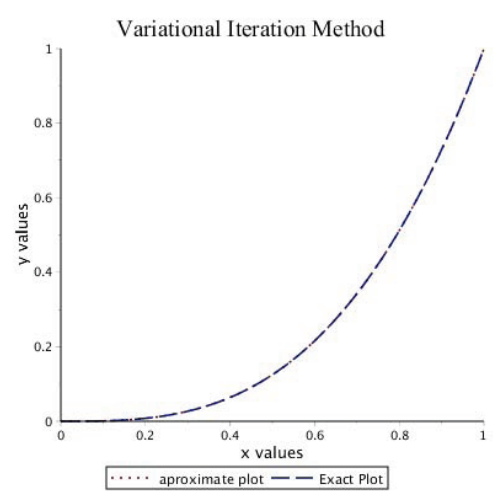

Figure 3. Comparison of the approximate solution by VIM with the exact solution

Table 3 shows the approximate solution for $n=3$, also it is obvious that we can improve the accuracy of solutions by computing more terms of approximate solutions. We can construct the following homotopy according to HPM,

$$
\begin{aligned}
\mathcal{H}(\mathrm{u}, p)= & (1-p)(\mathrm{u}(t)-\mathrm{g}(t))+p\left(\mathrm{u}(t)-\left(t^{3}-\frac{t^{19}}{100}-\frac{t^{20}}{110}\right)\right. \\
& \left.-\frac{t^{3}}{10} x^{2}(t) \int_{0}^{t}(s+1) x^{3}(s) d s\right)=0
\end{aligned}
$$

such that $\mathrm{g}(t)=\left(t^{3}-\frac{t^{19}}{100}-\frac{t^{20}}{110}\right)$, then

$$
\mathcal{H}(\mathrm{u}, p)=\mathrm{u}(t)-\left(t^{3}-\frac{t^{19}}{100}-\frac{t^{20}}{110}\right)-p \frac{t^{3}}{10} x^{2}(t) \int_{0}^{t}(s+1) x^{3}(s) d s=0
$$


substituting (11) into (29) and equating the terms with identical powers of $p$ we have

$$
\begin{aligned}
p^{0}: \mathrm{u}_{0}(t)= & t^{3}-\frac{t^{19}}{100}-\frac{t^{20}}{110} \\
p^{1}: \mathrm{u}_{1}(t)= & \frac{t^{3}}{10} A_{0}(t) \int_{0}^{t}(s+1) B_{0}(s) d s \\
p^{2}: \mathrm{u}_{2}(t)= & \frac{t^{3}}{10} A_{0}(t) \int_{0}^{t}(s+1) B_{1}(s) d s+\frac{t^{3}}{10} A_{1}(t) \int_{0}^{t}(s+1) B_{0}(s) d s \\
p^{3}: \mathrm{u}_{3}(t)= & \frac{t^{3}}{10} A_{0}(t) \int_{0}^{t}(s+1) B_{2}(s) d s+\frac{t^{3}}{10} A_{1}(t) \int_{0}^{t}(s+1) B_{1}(s) d s \\
& +\frac{t^{3}}{10} A_{2}(t) \int_{0}^{t}(s+1) B_{0}(s) d s
\end{aligned}
$$

and so on, where $A_{i}$ and $B_{i}$ are He's polynomials of the nonlinear terms $x^{2}$ and $x^{3}$ respectively, and the solution will be,

$$
\mathrm{u}(t)=\sum_{i=0}^{n} \mathrm{u}_{i}(t),
$$

Table 4. Comparison of the numerical results with the exact solution $x(t)$

\begin{tabular}{cccc}
\hline$t$ & Approximate Solution & Exact Solution & Absolute error \\
\hline 0.10 & 0.00100000 & 0.00100000 & $1 \times 10^{-73}$ \\
0.20 & 0.00800000 & 0.00800000 & $1.611 \times 10^{-53}$ \\
0.30 & 0.02700000 & 0.02700000 & $1.363 \times 10^{-41}$ \\
0.40 & 0.06400000 & 0.06400000 & $4.227 \times 10^{-33}$ \\
0.50 & 0.12500000 & 0.12500000 & $1.704 \times 10^{-26}$ \\
0.60 & 0.21600000 & 0.21600000 & $4.389 \times 10^{-21}$ \\
0.70 & 0.34300000 & 0.34300000 & $1.688 \times 10^{-16}$ \\
0.80 & 0.51200000 & 0.51200000 & $1.607 \times 10^{-12}$ \\
0.90 & 0.72899999 & 0.72900000 & $5.210 \times 10^{-9}$ \\
1.00 & 0.99999316 & 1.00000000 & 0.0000068386 \\
\hline
\end{tabular}

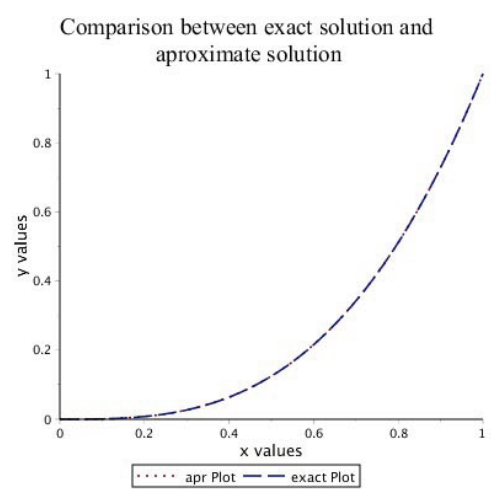

Figure 4. Comparison of the approximate solution by HPM with the exact solution

Table 4 shows the approximate solution for $n=3$, also it is obvious that we can improve the accuracy of solutions by computing more terms of approximate solutions. 
Example 3. Solve the QIE (Bana's et al., 2005)

$$
x(t)=t^{3}+\left(\frac{1}{4} x(t)+\frac{1}{4}\right)\left(\int_{0}^{t} t+\cos \left(\frac{x(s)}{1+x^{2}(s)}\right)\right) d s .
$$

According to the VIM, differentiating both sides of eq.(34) ones with respect to $t$ gives the IDE

$$
\begin{aligned}
x^{\prime}(t)= & 3 t^{2}+\frac{1}{2} t x(t)+\frac{1}{4} t^{2} x^{\prime}(t)+\frac{1}{4} x(t) \cos \left(\frac{x(t)}{1+x^{2}(t)}\right) \\
& +\frac{1}{4} x^{\prime}(t) \int_{0}^{t} \cos \left(\frac{x(s)}{1+x^{2}(s)}\right) d s+\frac{1}{2} t+\frac{1}{4} \cos \left(\frac{x(t)}{1+x^{2}(t)}\right),
\end{aligned}
$$

The correction functional for eq.(35) is

$$
\begin{aligned}
x_{n+1}(t)= & x_{n}(t)-\int_{0}^{t}\left(x_{n}^{\prime}(\zeta)-3 \zeta^{2}-\frac{1}{2} \zeta x_{n}(\zeta)-\frac{1}{4} \zeta^{2} x_{n}^{\prime}(\zeta)-\frac{1}{4} x_{n}(\zeta) \cos \left(\frac{x_{n}(\zeta)}{1+x_{n}^{2}(\zeta)}\right)\right. \\
& -\frac{1}{4} x_{n}^{\prime}(\zeta) \int_{0}^{\zeta} \cos \left(\frac{x_{n}(r)}{1+x_{n}^{2}(r)}\right) d r-\frac{1}{2} \zeta-\frac{1}{4} \cos \left(\frac{x_{n}(\zeta)}{1+x_{n}^{2}(\zeta)}\right) d \zeta
\end{aligned}
$$

we can use the initial value $x(0)=0$ to obtain the zeroth approximation $x_{0}(t)$ and by using the eq.(36) we get the successive approximations

$$
\begin{aligned}
x_{0}(t)= & 0, \\
x_{1}(t)= & \frac{1}{4} t+t^{3}+\frac{1}{4} t^{2}, \\
x_{2}(t)= & \frac{1}{4} t+\frac{431}{384} t^{3}+\frac{5}{16} t^{2}-\frac{1}{56} t^{10}-\frac{5}{336} t^{9}-\frac{71}{3360} t^{8}-\frac{1499}{53760} t^{7} \\
& -\frac{269}{15360} t^{6}+\frac{3599}{15360} t^{5}+\frac{473}{1536} t^{4},
\end{aligned}
$$

and so on, and the solution given by

$$
x(t)=\lim _{n \rightarrow \infty} x_{n}(t)
$$

We can construct the following homotopy according to HPM,

$$
\begin{gathered}
\mathcal{H}(\mathrm{u}, p)=(1-p)\left(\mathrm{u}(t)-t^{3}\right)+p\left(\mathrm{u}(t)-t^{3}-\left(\frac{1}{4} \mathrm{u}(t)+\frac{1}{4}\right) \int_{0}^{t} t+\cos \left(\frac{\mathrm{u}(s)}{1+\mathrm{u}^{2}(s)}\right) d s\right)=0, \\
\mathcal{H}(\mathrm{u}, p)=\mathrm{u}(t)-t^{3}-p\left(\frac{1}{4} \mathrm{u}(t)+\frac{1}{4}\right) \int_{0}^{t} t+\cos \left(\frac{\mathrm{u}(s)}{1+\mathrm{u}^{2}(s)}\right) d s=0,
\end{gathered}
$$


substituting (11) into (41) and equating the term with identical powers of $p$ we have

$$
\begin{aligned}
p^{0}: \mathrm{u}_{0}(t)= & t^{3}, \\
p^{1}: \mathrm{u}_{1}(t)= & \frac{1}{4} \mathrm{u}_{0}(t) \int_{0}^{t}\left(t+H_{0}(s)\right) d s+\frac{1}{4} \int_{0}^{t}\left(t+H_{0}(s)\right) d s, \\
p^{2}: \mathrm{u}_{2}(t)= & \frac{1}{4} \mathrm{u}_{0}(t) \int_{0}^{t} H_{1}(s) d s+\frac{1}{4} \int_{0}^{t} H_{1}(s) d s \\
& +\frac{1}{4} \mathrm{u}_{1}(t) \int_{0}^{t}\left(t+H_{0}(s)\right) d s, \\
p^{3}: \mathrm{u}_{3}(t)= & \frac{1}{4} \mathrm{u}_{0}(t) \int_{0}^{t} H_{2}(s) d s+\frac{1}{4} \int_{0}^{t} H_{2}(s) d s+\frac{1}{4} \mathrm{u}_{1}(t) \int_{0}^{t} H_{1}(s) d s \\
& +\frac{1}{4} \mathrm{u}_{2}(t) \int_{0}^{t}\left(t+H_{0}(s)\right) d s,
\end{aligned}
$$

and so on, where $H_{i}$ are He's polynomials of the nonlinear term $\cos \left(\frac{x(s)}{1+x^{2}(s)}\right)$ and the solution will be

$$
\mathrm{u}(t)=\sum_{i=0}^{n} \mathrm{u}_{i}(t),
$$

Table 5. Approximate solution $x(t)$ by VIM and HPM for $n=1$

\begin{tabular}{ccc}
\hline$t$ & VIM solution & HPM solution \\
\hline 0.10 & 0.02930184 & 0.02930216 \\
0.20 & 0.07228471 & 0.07229271 \\
0.30 & 0.13761286 & 0.13768016 \\
0.40 & 0.23575023 & 0.23610759 \\
0.50 & 0.37941576 & 0.38085479 \\
0.60 & 0.58374728 & 0.58851618 \\
0.70 & 0.86560555 & 0.87921321 \\
0.80 & 1.24089607 & 1.27522943 \\
0.90 & 1.71800041 & 1.79569813 \\
1.00 & 2.28475926 & 2.44303370 \\
\hline
\end{tabular}

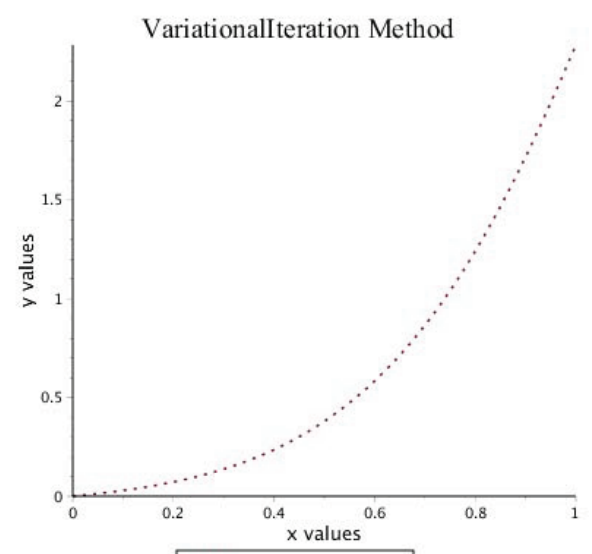

(a)

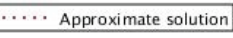

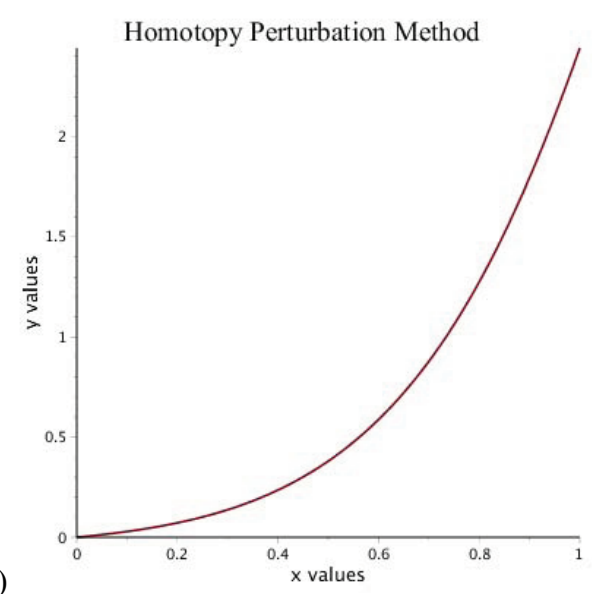

(b)

Figure 5. Approximate solutions by using (VIM) and (HPM) 
Example 4. Solve QIE (Bana's \& Martinon, 2004)

$$
x(t)=e^{-t}+x(t) \int_{0}^{t} \frac{t^{2} \ln (1+s|x(s)|)}{2 e^{(t+s)}} d s, \quad 0<t \leq 2 .
$$

According to VIM, differentiating both sides of Equation (46) ones with respect to $t$ gives the IDE

$$
\begin{aligned}
x^{\prime}(t)= & -e^{-t}+x^{\prime}(t) \int_{0}^{t} \frac{t^{2} \ln (1+s|x(s)|)}{2 e^{(t+s)}}+x(t)\left(\frac{t^{2}}{2 e^{2 t}} \ln (1+t|x(t)|)\right. \\
& \left.+\int_{0}^{t} \frac{-2 t^{2} e^{(t+s)}+4 t e^{(t+s)}}{4 e^{(t+s)^{2}}} \ln (1+s|x(s)|)\right), \quad x(0)=1 .
\end{aligned}
$$

The correction functional for eq.(47) is

$$
\begin{aligned}
x_{n+1}=x_{n}(t) & -\int_{0}^{t}\left(x_{n}^{\prime}(\zeta)+e^{-\zeta}-x_{n}^{\prime}(\zeta) \int_{0}^{\zeta} \frac{\zeta^{2}}{2 e^{(\zeta+r)}} \ln \left(1+r\left|x_{n}(r)\right|\right) d r\right. \\
& \left.-x_{n}(\zeta)\left[\frac{\zeta^{2}}{2 e^{2 \zeta}} \ln \left(1+\zeta\left|x_{n}(\zeta)\right|\right)+\int_{0}^{\zeta} \frac{\left.-2 \zeta^{2} e^{(\zeta+r)}+4 \zeta e^{(\zeta+r)}\right)}{4 e^{(\zeta+r) 2}} \ln \left(1+r\left|x_{n}(r)\right|\right) d r\right]\right) d \zeta
\end{aligned}
$$

the zeroth approximation $x_{0}(t)$ can be selected by using the initial value $x(0)=1$. We can construct the following homotopy according to HPM,

$$
\mathcal{H}(\mathrm{u}, p)=\mathrm{u}(t)-e^{-t}-p \mathrm{u}(t) \int_{0}^{t} \frac{t^{2} \ln (1+s|\mathrm{u}(s)|)}{2 e^{(t+s)}}=0,
$$

substituting (11) into (48) and equating the terms with identical powers of $p$ we have

$$
\begin{aligned}
& p^{0}: \mathrm{u}_{0}(t)=e^{-t}, \\
& p^{1}: \mathrm{u}_{1}(t)=\mathrm{u}_{0}(t) \int_{0}^{t} \frac{t^{2}}{2 e^{(t+s)}} H_{0}(s) d s, \\
& p^{2}: \mathrm{u}_{2}(t)=\mathrm{u}_{0}(t) \int_{0}^{t} \frac{t^{2}}{2 e^{(t+s)}} H_{1}(s) d s+\mathrm{u}_{1}(t) \int_{0}^{t} \frac{t^{2}}{2 e^{(t+s)}} H_{0}(s) d s,
\end{aligned}
$$

and so on, where $H_{i}$ are He's polynomials of the nonlinear term $\ln (1+s|x(s)|)$.

Table 6. Approximate solution $x(t)$ by VIM and HPM for $n=1$

\begin{tabular}{ccc}
\hline$t$ & VIM solution & HPM solution \\
\hline 0.10 & 0.90486075 & 0.90485481 \\
0.20 & 0.81907560 & 0.81892557 \\
0.30 & 0.74240511 & 0.74151336 \\
0.40 & 0.67478739 & 0.67187791 \\
0.50 & 0.61603248 & 0.60924288 \\
0.60 & 0.56560069 & 0.55284340 \\
0.70 & 0.52254959 & 0.50196629 \\
0.80 & 0.48565135 & 0.45597253 \\
0.90 & 0.45360274 & 0.41430435 \\
1.00 & 0.42522512 & 0.37648238 \\
\hline
\end{tabular}


(c)

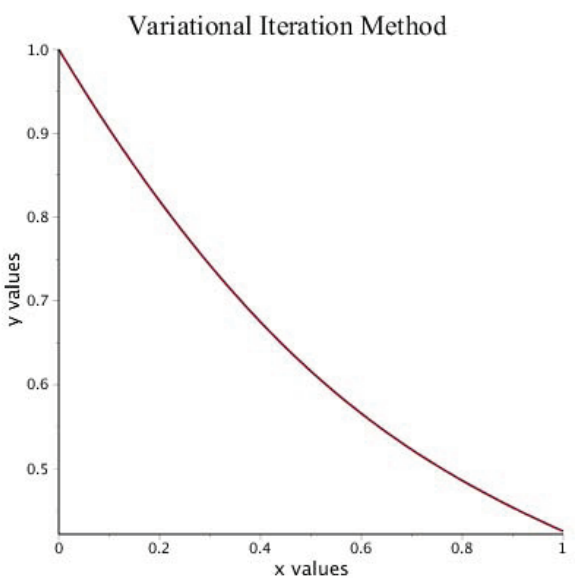

Figure 6. Approximate solution by using (VIM) and (HPM)

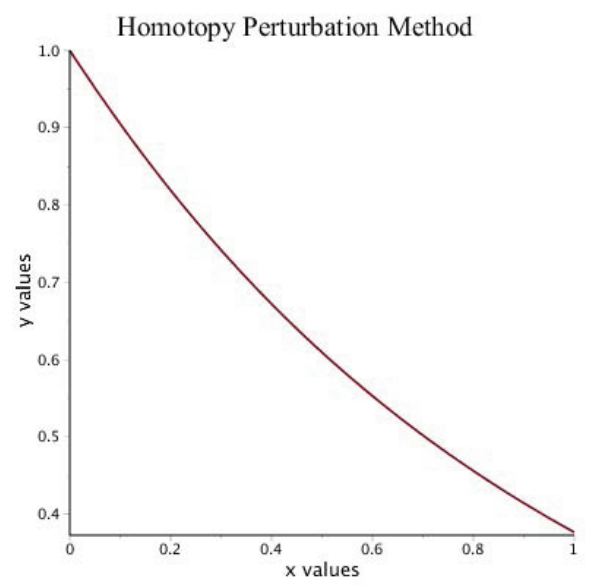

d)

\section{Conclusion}

We have been successfully applied the VIM and HPM to find the approximate solutions for nonlinear QIEs. We have found out that the two methods are applicable and efficient technique, also the HPM is better than VIM in finding the accurate solutions. We have been observing that the accuracy can be improved by computing more n-terms off approximate solutions or by taking more terms in the Taylor expansion of the nonlinear terms. To find the calculations we have used the Maple package (2015).

\section{References}

Abdou, M. A., \& Soliman, A. A. (2005). Variational iteration method for solving Burger's and coupled Burger's equations. Journal of Computational and Applied Mathematics, 181(2), 245-251. https://doi.org/10.1016/j.cam.2004.11.032

Abulwafa, E. M., Abdou, M. A., \& Mahmoud, A. A. (2006). The solution of nonlinear coagulation problem with mass loss. Chaos, Solitons E Fractals, 29(2), 313-330. https://doi.org/10.1016/j.chaos.2005.08.044

Argyros, I. K. (1985). Quadratic equations and applications to Chandrasekhar's and related equations. Bulletin of the Australian Mathematical Society, 32(02), 275-292. https://doi.org/10.1017/S0004972700009953

Avazzadeh, Z. (2012). A Numerical Approach for Solving Quadratic Integral Equations of Urysohn's Type using Radial Basis Function. Journal of Applied E Computational Mathematics, 1(4).

Banaś, J., Caballero, J., Rocha, J., \& Sadarangani, K. (2005). Monotonic solutions of a class of quadratic integral equations of Volterra type. Computers $\mathcal{G}$ Mathematics with Applications, 49(5), 943-952. https://doi.org/10.1016/j.camwa.2003.11.001

Banaś, J., Lecko, M., \& El-Sayed, W. G. (1998). Existence theorems for some quadratic integral equations. Journal of Mathematical Analysis and Applications, 222(1), 276-285. https://doi.org/10.1006/jmaa.1998.5941

Banaś, J., \& Martinon, A. (2004). Monotonic solutions of a quadratic integral equation of Volterra type. Computers $\mathcal{E}$ Mathematics with Applications, 47(2), 271-279. https://doi.org/10.1016/S0898-1221(04)90024-7

El-Sayed, A. M. A., \& Hashem, H. H. G. (2009a). Monotonic solutions of functional integral and differential equations of fractional order. Electronic Journal of qualitative theory of differential equations, (7), 1-8.

El-Sayed, A. M. A., \& Hashem, H. H. G. (2009b). Solvability of nonlinear Hammerstein quadratic integral equations. Journal of Nonlinear Sciences $\mathcal{E}$ its Applications, 2(3), 152-160.

El-Sayed, A. M. A., Hashem, H. H. G., \& Ziada, E. A. A. (2010). Picard and Adomian methods for quadratic integral equation. Computational $\mathcal{F}$ Applied Mathematics, 29(3), 447-463. https://doi.org/10.1590/S1807-03022010000300007

El-Sayed, W. G., \& Rzepka, B. (2006). Nondecreasing solutions of a quadratic integral equation of Urysohn type. Computers $\mathcal{G}$ Mathematics with Applications, 51(6), 1065-1074. https://doi.org/10.1016/j.camwa.2005.08.033

He, J. H. (1999a). Homotopy perturbation technique. Computer methods in applied mechanics and engineering, 178(3), 257-262. https://doi.org/10.1016/S0045-7825(99)00018-3 
He, J. H. (1999b). Variational iteration methodCa kind of non-linear analytical technique: some examples. International journal of non-linear mechanics, 34(4), 699-708. https://doi.org/10.1016/S0020-7462(98)00048-1

He, J. H. (2000). A coupling method of a homotopy technique and a perturbation technique for non-linear problems. International journal of non-linear mechanics, 35(1), 37-43. https://doi.org/10.1016/S0020-7462(98)00085-7

He, J. H. (2003). Homotopy perturbation method: a new nonlinear analytical technique. Applied Mathematics and computation, 135(1), 73-79. https://doi.org/10.1016/S0096-3003(01)00312-5

He, J. H. \& Wu, X. H. (2007). Variational iteration method: new development and applications. Computers $\mathcal{E}$ Mathematics with Applications, 54(7), 881-894. https://doi.org/10.1016/j.camwa.2006.12.083

Liao, S. J. (1995). An approximate solution technique not depending on small parameters: a special example. International Journal of Non-Linear Mechanics, 30(3), 371-380. https://doi.org/10.1016/0020-7462(94)00054-E

Wazwaz, A. M. (2007a). The variational iteration method: A reliable analytic tool for solving linear and nonlinear wave equations. Computers $\mathcal{E}$ Mathematics with Applications, 54(7), 926-932.

https://doi.org/10.1016/j.camwa.2006.12.038

\section{Copyrights}

Copyright for this article is retained by the author(s), with first publication rights granted to the journal.

This is an open-access article distributed under the terms and conditions of the Creative Commons Attribution license (http://creativecommons.org/licenses/by/4.0/). 